\title{
X-ray photon-in/photon-out methods for chemical imaging
}

\author{
Matthew A. Marcus
}

\begin{abstract}
Most interesting materials in nature are heterogeneous, so it is useful to have analytical techniques with spatial resolution sufficient to resolve these heterogeneities. This article presents the basics of X-ray photon-in/photon-out chemical imaging. This family of methods allows one to derive images reflecting the chemical state of a given element in a complex sample, at micron or deep sub-micron scale. $X$-ray chemical imaging is relatively non-destructive and element-selective, and requires minimal sample preparation. The article presents the basic concepts and some considerations of data taking and data analysis, along with some examples.
\end{abstract}

Keywords: Chemical imaging; Data analysis; Element-selective; Microprobe; NEXAFS; Microdiffraction; Non-destructive; Valence-state imaging; XANES; X-ray fluorescence

Matthew A. Marcus* The Advanced Light Source, Lawrence Berkeley National Laboratory, 1 Cyclotron Road, Berkeley, CA 94720, USA

"Tel.: +1 510495 2106; E-mail: mamarcus@lbl.gov

\section{Introduction}

Almost all interesting materials are heterogeneous. This heterogeneity can take many forms, of which one is that of chemical state. For example, in the environmental field, $\mathrm{Cr}$ is a common pollutant that is far more toxic in hexavalent form than trivalent [1]. The toxicity of As depends not only on its valence but also whether it is in organo-As or inorganic form [2]. The oxidation state of redox-active elements can also yield information about the conditions under which the material of interest was formed. It can therefore be useful to have a way of mapping the chemical state of a given element (i.e. its valence or environment), so as to be able to correlate it with other features of the sample and so understand why it is where it is and in the form that it is in. For example, for redox-active species, one might want to know if the presence of some other species causes reduction or oxidation.

X-ray methods can be very useful in this endeavor. X-rays are minimally destructive and can be formed into nm-scale probes. They generally penetrate more deeply than electrons, so sample preparation can be easier and less invasive than for electron-based methods. X-ray fluorescence can be used to detect trace elements at parts per million (ppm) levels, which exceed the typical sensitivities of particlebeam methods. The energy position and shape of absorption edges is sensitive to chemical state (valence and coordination geometry) and X-ray diffraction responds to crystal structure. The basics of the use of synchrotron light sources to perform such measurements are nicely covered in a review by Lombi and Susini [3].

Finally, the increasing availability of synchrotron light sources has made it practical to perform analyses that would have been difficult to impossible in previous decades. For all these reasons, X-ray methods, particularly those in which both incident probe and received signals are Xray photons, have become important and popular analytic tools.

In this article, I show how hard X-rays $(\mathrm{E}>1 \mathrm{keV})$ can be used for element-specific, chemically-sensitive imaging, with illustrative examples and some discussion of theory.

Most of the work in this area has been done with two-dimensional mapping, but there have been significant efforts in which three-dimensional (tomographic) methods have been employed \{e.g., Golosio et al. [4] (tomography), and Vincze et al. [5] and Vekemans et al. [6] (confocal microscopy)\}. The contrast mechanisms and the basic concepts are common to both sets of studies, so what is said about two-dimensional imaging mostly carries over into the third dimension.

\subsection{Contrast mechanisms}

I will discuss two mechanisms by which chemical state can be translated into contrast. The first is that used in diffraction mapping. In this method, an X-ray 
beam is rastered over the sample and an X-ray diffraction pattern collected at each pixel using a 2D detector (e.g., a CCD). Phases present as powders with grain size much smaller than the probe beam yield rings (Debye rings), whose radii depend on the $d$-spacings of the strong reflections from those phases. By azimuthal integration of the Debye rings in patterns taken at each point, one can make a 2D map of the abundance of each phase as a function of position in the sample. While this method does not directly yield chemical-state information, the identification of specific phases implies identification of at least one aspect of the chemical state of the major elements in that phase. For example, if goethite is the only mineral found at a point and there are no amorphous phases, then the chemistry of $\mathrm{Fe}$ at that point is uniquely defined. This technique was used in conjunction with X-ray fluorescence (XRF) mapping by Manceau et al. to study incorporation of trace elements in natural nanophase oxides in soil ferromanganese nodules [7]. Dooryhée et al. [8] investigated a Roman painting to determine what pigments were used and how their grain sizes correlated with the visual appearance of the artwork. This technique is often referred to as mineral mapping.

The more-widely used form of chemical imaging relies on the effects of chemical state on the X-ray absorption spectrum from a given element. While this method was pioneered by Kinney et al. (e.g., [9,10]) in the 1980s, it could be argued that Pickering's 2000 paper [11] gave the clearest exposition of data analysis and quantitation in real, as opposed to model, samples. As shown in the top panels of Fig. 1 [12] and Fig. 2 [13], the absorption spectrum from $\mathrm{Fe}$, at both K-edge and L-edge, depends on its valence as well as other structural properties. By mapping absorption, using either transmission or fluorescence detection, at several energies (shown as vertical lines in Fig. 1), one can acquire what is essentially an X-ray absorption near-edge structure (XANES) spectrum at each pixel or voxel [14] and fit this to a sum of standard spectra.

This kind of chemical mapping has several interesting features. It is element selective, so that the chemistry of trace elements may be probed, even in the presence of much larger quantities of other elements. Although radiation damage can be an issue, X-ray methods tend to be less damaging than transmission-electron methods (e.g., electron energy loss spectroscopic imaging). When hard X-rays are used, the probe depth is typically microns or greater, allowing probing of buried layers (e.g., Fig. 1 shows a sample embedded in an aerogel block, which would be hard to probe with most other methods). Sample preparation is generally minimal, so, when planning a campaign of analysis on a precious sample, X-ray microprobe methods, including chemical mapping, can come early in the chain, before the most destructive sample preparation and analysis procedures.
In the soft X-ray range, the K-edge spectra of light elements (e.g., $\mathrm{C}, \mathrm{N}$ and $\mathrm{O}$ ) exhibit rich structureencoding information about functional groups as well as valence [15] (e.g., at the $\mathrm{C} \mathrm{K}$-edge, there are features at specific energies associated with transitions into $\mathrm{C}-\mathrm{C}$ and $\mathrm{C}-\mathrm{O} \sigma^{*}$ and $\pi^{*}$ peaks, with the exact energies depending on what functional groups the $\mathrm{C}$ atom is bonded to). Similar effects can be used at $\mathrm{O}$ and $\mathrm{N}$ edges to detect protein and DNA, thus enabling the imaging of cells in a way that highlights the biochemistry [13,16-18] and also allowing visualization of minerals through L-edge absorption of elements (e.g., Ca and Fe).

\section{Examples}

A few examples provide a general impression of what can be done with these methods and how they complement other techniques. In these examples, I present speciation maps without explanation of how they were derived from raw data. The theory of data analysis is discussed in the next section, and some of the practicalities of data collection in the section after that.

The bottom panel of Fig. 1 shows a chemical map on a track from the Stardust cometary collector [12]. The data for this map was collected at ALS (Advanced Light Source) Beamline 10.3.2 at the energies shown in the top panel of Fig. 1. The spectra used to produce the map are K-edge spectra of mixtures of species, weighted according to their abundances in a large sample of Stardust tracks. These species are grouped into metallic (including carbide), sulfide, $\mathrm{Fe}^{2+}$ and $\mathrm{Fe}^{3+}$ phases. Not only is there a diverse set of species, but there is also large-scale variation in the chemistry across the track. Since this track was produced by the capture of a single particle from the comet into aerogel, the spatial segregation might mean that the particle contained regions of different composition. In other words, the particle was a micron-size rock comprising several minerals in discrete locations, as opposed to being a fine-grained mixture.

Chemical mapping has been used for the study of As, S and Se in plants (e.g., Bulska et al. [19], Sutton et al. [20] and Pickering et al [21-23]). Reviews on this topic have been published recently by Lombi and Susini [3] and Thieme [24].

The toxicity of Se and As depends on their chemical form, and the biochemical pathways leading to the uptake of these elements may be identified in part by determining the final form of the element. A leaf of Astragalus bisulcatus plants grown in selenate-enriched soil is covered with trichomes rich in selenate, while the rest of the leaf contains $\mathrm{Se}$ in the form of methylselenocysteine [25]. Freeman et al.'s hypothesis is that the organic form is less toxic to the plant than other species, so serves as a "safe" storage form, and the presentation 

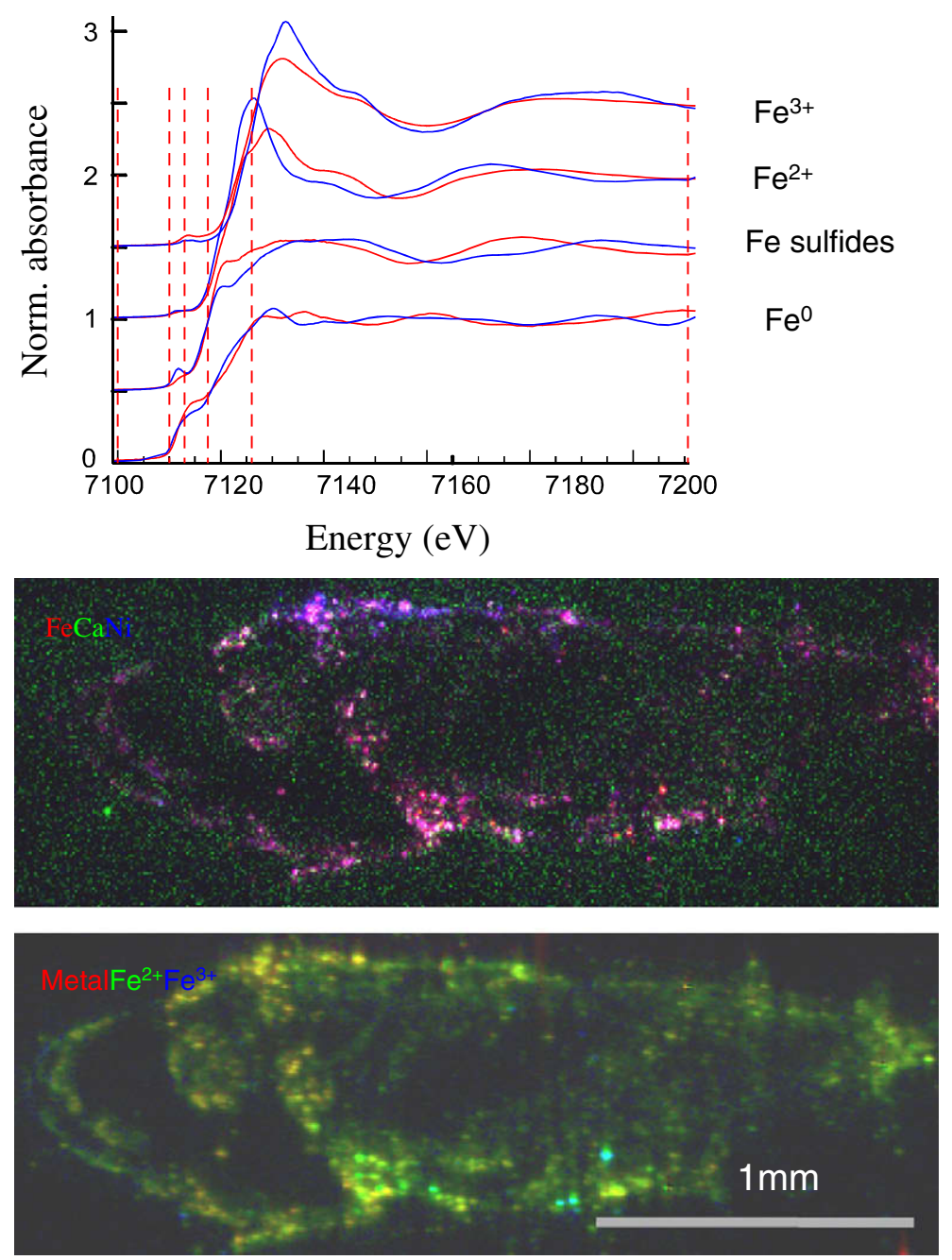

Figure 1. (Top) XANES spectra for Fe in four different chemical states ( $\mathrm{Fe}^{0}, \mathrm{Fe}^{2}+, \mathrm{Fe}^{3+}$ and $\mathrm{Fe}$ sulfides), each represented by two minerals (shown in red and heavier blue lines.). The minerals are: (in red) awaruite ( $\mathrm{FCC} \mathrm{Fe}: \mathrm{Ni})$, pyrrhotite ( $\mathrm{FeS}$ ), augite $\left(\left(\mathrm{Fe}^{2+}, \mathrm{Mg}, \mathrm{Ca}\right)_{2} \mathrm{Si}_{2} \mathrm{O}_{6}\right)$ and ferrihydrite $\left(\mathrm{Fe}^{3+} \mathrm{OOH}\right)$; and, (n blue) kamacite $\left(\mathrm{BCC} \mathrm{Ni}: \mathrm{Fe}^{0}\right)$, pentlandite $\left(\left(\mathrm{Fe}, \mathrm{Ni}_{9} \mathrm{~S}_{8}\right)\right.$, fayalite $\left(\mathrm{Fe}^{2+}{ }_{2} \mathrm{SiO}_{4}\right)$ and $\mathrm{Fe}^{3+}$-bearing smectite clay. These references are as used in [51]. The dashed vertical lines indicate the energies at which images were taken to produce the chemical image at the bottom of this Figure.

(Middle) XRF map of a Stardust comet-particle track showing Fe as red, $\mathrm{Ca}$ in green and $\mathrm{Ni}$ in blue. The variously-colored spots represent fragments of varying chemical composition.

(Bottom) Chemical image of the same sample showing $\mathrm{Fe}^{0}, \mathrm{Fe}^{2+}$ and $\mathrm{Fe}^{3+}$ in red, green and blue, respectively. The maps were done with a $20 \mu \mathrm{m}$ pixel; the scale bar is $1 \mathrm{~mm}$.

of the highly-toxic selenate on the leaf surface deters herbivory.

Chemical mapping also finds application in the environmental and geological sciences. An example, using soft X-rays at ALS Beamlines 5.3.2 and 11.0.2, is an investigation by Toner et al. [13] of the state of Fe in particles in the plume of a mid-ocean hydrothermal vent. The top part of Fig. 2 shows the L-edge spectra for Fe in ferrihydrite (hydrous ferric oxide), pyrrhotite $(\sim \mathrm{FeS})$ and three areas on a sample. The vertical bars show the positions of characteristic peaks for $\mathrm{Fe}^{2+}$ and $\mathrm{Fe}^{3+}$, the positions of which are nearly independent of the structure of the host material. The unknown spectra clearly show the presence of both valence states, but the spectra cannot be simulated as a combination of ferrihydrite (detected by hard X-ray XANES) and pyrrotite or any of a number of other ferrous minerals. Instead, the spectra are consistent with Fe complexed to organic ligands. The chemical maps in the bottom part of Fig. 2 were derived by taking stacks (sequences of images at varying energies) and using singular-value decomposition and fitting methods to derive signals representing $\mathrm{Fe}^{2+}$ and $\mathrm{Fe}^{3+}$. We see that the $\mathrm{Fe}^{2+}$ is mostly in areas high in carbon. Further, spectroscopy on the carbon K-edge shows distinct signatures of biomolecules (e.g., proteins, lipids and polysaccharides). The inference, supported by other lines 

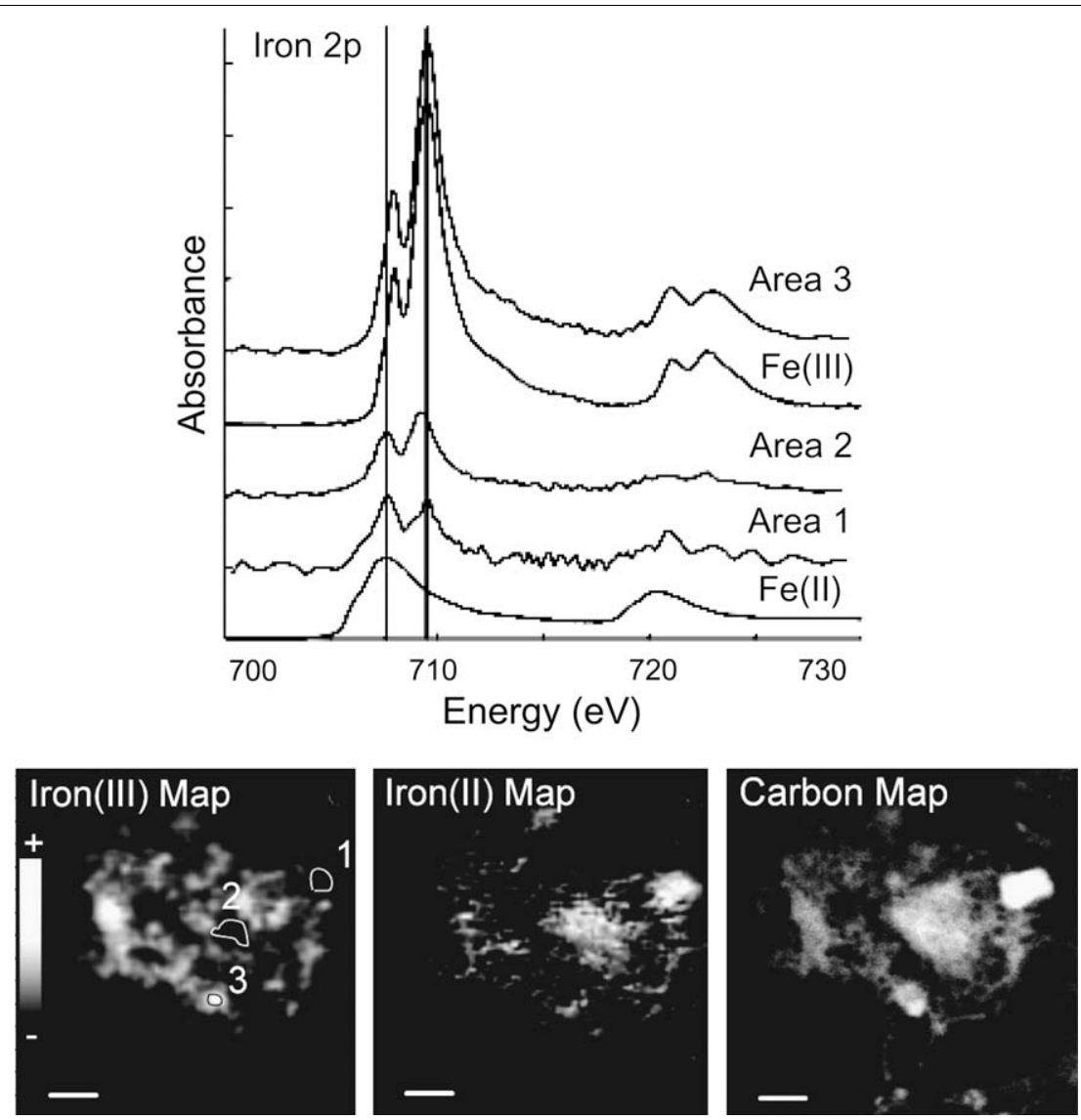

Figure 2. (Top) XANES spectra at the Fe L-edge for ferrihydrite $\left(\mathrm{Fe}^{3+}\right)$, pyrrhotite and three parts of a complex hydrothermal-plume particle. Vertical bars show the peak positions characteristic of $\mathrm{Fe}^{2+}$ and $\mathrm{Fe}^{3+}$.

(Bottom) Chemical-state-specific images for $\mathrm{Fe}^{2+}$ and $\mathrm{Fe}^{3+}$ and an image of total carbon. Labels correspond to the areas (bounded by white curves) represented by the XANES spectra above. Scalebars are $1 \mu \mathrm{m}$.

of evidence, is that the $\mathrm{Fe}^{2+}$ is protected from rapid oxidation by dissolved oxygen in the seawater by being bound up in organic materials that were probably made by microbes. This preservation provides a means for reduced Fe (the bioavailable form) to be transported over long distances, thus feeding into the global Fe cycle.

Chrome-ore-processing residue (COPR) is a byproduct of the Cr-mining industry that has been used as landfill. COPR contains significant amounts of hexavalent $\mathrm{Cr}$, which can contaminate surrounding areas. Chrysochoou et al. [26] imaged the distribution of $\mathrm{Cr}^{6+}$ in COPR as part of an effort to understand the physical and chemical forms that $\mathrm{Cr}^{6+}$ takes and the connection between the phase distribution and the effectiveness of remediation by chemical reduction [27]. The top half of Fig. 3 shows the $\mathrm{Ca}$ and $\mathrm{Cr}^{6+}$ contents in two types of COPR - Gray-black (GB, unhydrated and loosely consolidated) and Hard brown (HB, bound with Ca-rich cementitious material). The cementing phase in $\mathrm{HB}$ does not show $\mathrm{Cr}^{6+}$, while the GB shows $\mathrm{Cr}^{6+}$ both in the larger "rocks" and the smaller particles between the large ones. The bottom half shows the $\mathrm{Cr}^{6+}$ contents of
GB before and after treatment with two reductants, calcium polysulfide and ferrous sulfate. One can see that the overall level of $\mathrm{Cr}^{6+}$ is reduced, but by no means rendered undetectable, and that, for both treatments, the highly-concentrated $\mathrm{Cr}^{6+}$-bearing phases lost $\mathrm{Cr}^{6+}$, while the matrix between particles and the less-concentrated particles themselves retained the toxic metal.

The dispersive-EXAFS beamline at the European Synchrotron Radiation Facility (ESRF) has been used to perform maps in which a complete XANES spectrum is collected at each pixel $[28,29]$, similar to what has been done with scanning transmission X-ray microscopy (STXM, see below). This technique was used to study the formation of serpentine minerals.

Foriel et al. [30] examined bacterial microfossils and living bacterial filaments and found similar patterns of $\mathrm{S}$ speciation, leading them to suggest that S-valence mapping might be used to detect biomarkers, thus helping to distinguish microfossils from look-alike abiotic structures. The S-chemical-state mapping was combined with XRF mapping of other elements and with synchrotron infrared microspectroscopy in order 

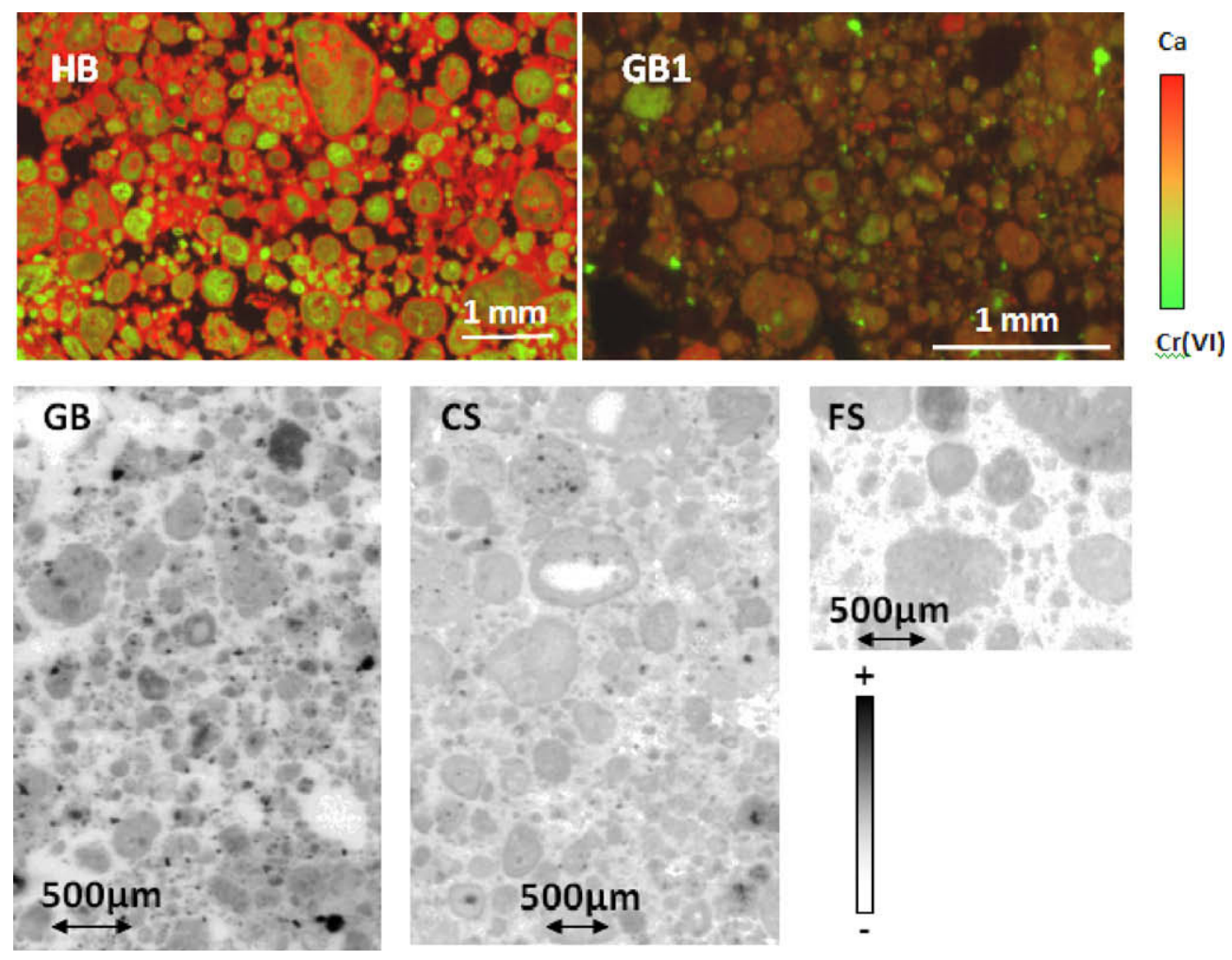

Figure 3. (Top) Bicolor-coded $\mu$ XRF maps showing $\mathrm{Ca}$ (in red) and $\mathrm{Cr}^{6+}$ (green) distributions in COPR HB and GB areas. Note the differing distributions of Ca-bearing cementitious material (red coatings) and $\mathrm{Cr}^{6+}$ (green).

(Bottom) Effect of two remediation treatments (calcium polysulfide, CS and ferrous sulfate, FS) on the amount and distribution of $\mathrm{Cr}^{6+}$ in GB-type COPR. The maximum display level for $\mathrm{Cr}^{6+}$ and $\mathrm{Ca}$ is the same between left and right images (top) and left, center and right images (bottom). The grayscale images are shown in negative contrast so that darker $=$ more $\mathrm{Cr}^{6+}$.

to obtain a fuller picture of the chemistry of their samples.

Strawn's group has used chemical mapping to study the speciation of Se in river sediment [31] and shale [32]. In a reclaimed mine soil, which originally contained Se as selenide minerals, they found an Fe-rich particle that had sorbed selenite, on which were smaller selenide particles. The observation of the small reduced particles showed that some of these original selenide minerals still existed, though most had weathered with release of selenite, which was subsequently sorbed onto Fe oxides. Without an imaging method, such a level of description would have been difficult to achieve.

\section{Data analysis}

While the basic concepts of chemical imaging are simple, there are subtleties to the analysis of the data. For diffraction mapping, the usual procedure is simply to do background subtraction and integration to find the intensities of the observed powder-diffraction rings that can be assigned to known phases. This simple procedure may incur artifacts due to variables (e.g., preferred ori- entation, self-absorption that causes the probed depth to vary with local composition, and grain size). The grain size comes into it because there may be only a small number of grains under the probe beam at any one time, so that the diffracted intensity at each pixel depends on the orientations of the grains that happen to be illuminated at that spot. Thus, diffraction mapping is most applicable to fine-grained materials.

For XANES mapping, the set of fluorescence or absorption maps or tomographs can be thought of as sets of XANES spectra, one per pixel or voxel. When acquiring data in fluorescence mode, the data from each pixel comprises either an energy spectrum of the X-rays emitted by the sample at that pixel or a set of sums of counts within energy bands covering the fluorescence peaks of the elements of interest. These "ROI sums" are read as proportional to the absorption signal from the given elements. If full spectra are taken, they can be analyzed to extract the relevant signals (e.g., using the PyMCA program [33]). In the hard X-ray regime, one typically takes only a few images, so that these "spectra" are tabulated at only a few energies. With the rapid imaging available using scanning transmission X-ray microscopy (STXM) or full-field transmission X-ray 
microscopy (TXM), it is possible to create "stacks", in which each pixel is represented by a full spectrum taken at hundreds of energies. In either case, the abundances of various species at each pixel are extracted by leastsquares fitting to sums of the spectra of reference materials. In addition, there is usually a background contribution such that the signal is non-zero at energies below the absorption edge. Thus, if one is looking for $N$ species, it is usually necessary to take images at least $N+1$ energies, one of which should be just below the absorption edge. The need for a background energy is especially great if one is imaging in transmission mode. In that mode, it is useful to include in the field of view a "blank" part of the sample, whose transmission spectrum can be subtracted off or used as a "species" in the fitting process.

If data are taken in fluorescence mode, then what one is doing is XRF at several energies, so the same sort of matrix effects and re-fluorescence corrections may apply as are discussed for XRF mapping in the article by Janssens, Vekemans and Vinzce elsewhere in this issue. More discussion of micro XRF mapping may be found in the article by Sutton et al. [34], who also discuss chemical mapping and tomography.

Absent artifacts, as described below in the section on practical considerations, the signal from each pixel may be described as a sum of the signals from all the assumed species:

$I(x, y, E)=S \sum_{i=1}^{N} c_{i}(x, y) Y_{i}(E)$

where $I(x, y, E)$ is the signal from the pixel at $x, y$ at energy $E, S$ is an overall sensitivity factor, $c_{i}(x, y)$ is the concentration of species $i$ at $x, y$, and $Y_{i}(E)$ is the normalized signal (usually taken as oscillating about 1 in the post-edge) for species $i$ at energy $E$. In fluorescence, $I$ is the fluorescence count rate divided by the incidentbeam intensity, whereas, in transmission, $I$ is the optical density $\left(\ln \left(I_{0} / I_{\text {trans }}\right)\right)$ with $I_{0}$ and $I_{\text {trans }}$ the incident and transmitted intensities. These definitions are exactly like those used in laboratory spectrophotometry. If a background needs to be subtracted, it can be included as a "species", for which $Y(E)=1$ for all $E$, or $Y_{b k d}(E)$ may be taken as a measured blank-area background. If one of the energies at which data are taken is significantly above the edge, then $Y_{i}(E) \cong 1$ for all non-background species, and so the image taken at that energy, after background subtraction, represents the total concentration of that element.

If there are only two species to be considered, then a simplified method of data acquisition and analysis may be used. In this method, data are taken at three energies:

- a pre-edge "background" energy;

- an energy relatively far above the edge so that $Y_{i}(E) \cong 1$; and,
- an intermediate energy at which the two species yield different signals.

Let these energies be labeled $E_{b k d}, E_{p o s t}$ and $E_{1}$, respectively. Then, we have:

$$
\begin{aligned}
& I\left(E_{1}\right)-I\left(E_{b k d}\right)=S\left(c_{1} Y_{1}\left(E_{1}\right)+c_{2} Y_{2}\left(E_{1}\right)\right) \\
& I\left(E_{p o s t}\right)-I\left(E_{b k d}\right)=S\left(c_{1}+c_{2}\right)
\end{aligned}
$$

where the $x, y$ arguments have been suppressed and it is assumed that the post-edge normalized signals from both species are 1. The indices 1,2 refer to the two species. Solving the simultaneous equations, we get:

$$
\begin{aligned}
& c_{1} S=\frac{\left(I^{\prime}\left(E_{1}\right)-Y_{2}\left(E_{1}\right) I^{\prime}\left(E_{2}\right)\right)}{Y_{1}\left(E_{1}\right)-Y_{2}\left(E_{1}\right)} \\
& c_{2} S=\frac{\left(I^{\prime}\left(E_{1}\right)-Y_{1}\left(E_{1}\right) I^{\prime}\left(E_{2}\right)\right)}{Y_{2}\left(E_{1}\right)-Y_{1}\left(E_{1}\right)}
\end{aligned}
$$

where $I^{\prime}(E)=I(E)-I\left(E_{b k d}\right)$ is the background-subtracted intensity at energy $E$. The sensitivity factor can either be ignored, in which case one gets images showing relative concentrations, or measured using a known standard. This factor is actually a slowly-varying function of energy, a complication that does not usually cause much error if ignored. For qualitative work, it is often sufficient to make a bicolor image with $I^{\prime}\left(E_{1}\right)$ and $I^{\prime}\left(E_{2}\right)$ assigned to two colors. Differences in chemistry then translate to differences in hue. This was the method adopted by Chrysochoou et al. [26] and is illustrated in Fig. 3.

If least-squares fitting is performed on a data set that includes images taken at more than $N+1$ energies, then the mean-square error at each pixel may be evaluated as an indication of the validity of the assumptions made in data analysis. Poor fits may result from noise, low concentrations of the element sought, over-absorption (the effect often mis-called "self-absorption") [35] or the presence of a species that yields a XANES spectrum unlike any of those used in the fit.

The result of these manipulations is a set of images showing the concentrations (relative or absolute) of the various species (valence states or, for light elements, functional groups). In addition, the set of images may include the strength of the background signal and the fitting error at each point.

The concentrations of three or fewer species may be displayed in a bicolor or tricolor map (e.g., the one on the bottom of Fig. 1). Such maps are often easy to read and understand on a qualitative level. All the other techniques by which XRF maps and similar spectral images can be displayed and understood may be applicable to such chemical maps.

In some cases, especially in the soft X-ray region, it is possible to take images at many energies so that each pixel represents a full XANES spectrum rather than a tiny abstract of one. In that case, each pixel contains more data values than the expected number of species so 
the analysis problem becomes in effect over-determined. Such over-determined problems are a perfect ground for analysis techniques based on singular-value decomposition (SVD). In one such technique, the spectra for all the pixels are expressed as sums of a small number of component spectra, derived solely by analysis of the data without reference to standard spectra. This is essentially a form of lossy data compression, in which the hundreds of data values per pixel are replaced by a smaller number of component loadings. Then, one can plot component strengths as colors in a bicolor or tricolor map, thus coloring by chemistry. Also, the pixels may be thought of as points in a high-dimensional space, which is projected to a lower dimension by SVD methods. These points may be grouped into clusters by automatic-classification algorithms, thus assigning a chemical type to each pixel. By plotting an image with each pixel rendered in a color depending on the chemical type to which it is assigned, a multicolored image may be made in which several species may be identified by a color code. This style of data analysis is described in some detail by Lerotic et al. [36]. Similar methods were used to produce the maps in the bottom half of Fig. 2.

\section{Practical considerations}

\subsection{Acquisition of data}

The most common method for chemical mapping is to use micro X-ray fluorescence mapping [37], as described in other articles in this issue, done at several energies. This technique is typically performed at microfocus $\mathrm{X}$-ray beamlines that use Kirkpatrick-Baez mirrors or zone plates to obtain a monochromatic micron-size or submicron-size beam. Examples of such beamlines include 10.3.2 at the Advanced Light Source [38] and 2ID-B at the Advanced Photon Source [39,40]. This method, popular in the hard X-ray regime, results in a number of maps, each showing the spatial distribution of the target element as seen by a probe X-ray beam at a given energy. Each of these maps may also display the distributions of other elements taken at the same time. In the hard X-ray regime, available resolutions range from the tens of $\mathrm{nm}$ to tens of microns, with $2-5 \mu \mathrm{m}$ being typical for synchrotron beamlines. Data may be taken in fluorescence mode or transmission. Fluorescence mode allows element selectivity, detection of trace elements, and use of thick samples. Transmission is useful for samples concentrated in the target element that can be made into thin sections (tens of microns). Adding a rotational degree of freedom to the sample stage allows the possibility of pencil-beam tomography (e.g., fluorescence tomography).

In the soft X-ray range, extending up to a few $\mathrm{keV}$ ("tender" X-rays), zone-plate-based scanning-probe instruments are very effective, usually operating in transmission mode (STXM). These devices yield spatial resolution down to $15 \mathrm{~nm}$. Fluorescence and total-electron yield detection are also possible, though technically challenging.

Another approach to image acquisition is full-field transmission imaging. In the hard X-ray regime, this is the most common mechanism for tomographic imaging, while projection microscopes have been useful in the soft X-ray range [41]. Except as explained below, the practical aspects of gathering data for chemical maps are the same as those for any type of X-ray imaging.

\subsection{Minimization of artifacts}

As with any experimental technique, there are a number of practical points that should be attended to in order to acquire useful data. One of these is the issue of sample or beamline drift. Many samples (e.g., leaves) cannot be mounted rigidly. Some change shape slightly during scanning. Thus, corresponding pixels on maps taken at different energies may not match up to the same point in the sample. If there are sharp gradients of composition (e.g., due to the presence of small particles), the error caused by subtracting data from mismatched locations will appear as "color fringes" in maps. Thus, particles containing only $\mathrm{Fe}^{3+}$ may appear to be coated on one side with $\mathrm{Fe}^{2+}$.

There are a few ways to deal with this problem. One, used in both hard X-ray and soft X-ray mapping (e.g., see the alignment feature of the AXIS2000 software of Hitchcock, et al. [42]), is to register all maps together (i.e., to distort the maps so that corresponding features match to each other). This task can often be done by using as fiducials features in the distributions of elements other than the target element, for which all the maps should look similar. The simplest sort of registration is done by shifting rigidly one map relative to another. However, the shift often varies as a function of time during the acquisition of each map, so that maps need to be "sheared" relative to each other. Thus, the displacement vector for each pixel is a function of the line number. Another effective approach, taken at beamline 2-3 at SSRL [43] [Web ref. 1], is to scan each line at every energy, rather than to scan the entire mapped area at one energy then switch to the next. This approach minimizes the time between acquisitions of a given pixel at successive energies.

Over-absorption can be a problem in fluorescencemode data collection. Consider, for example, a sample containing only the oxidized Fe species whose XANES spectra are shown in Fig. 1. A thick, concentrated spot will yield a spectrum in which over-absorption reduces the intensity above the edge while not attenuating the pre-edge signal. This effect results in a pre-edge signal that is greater relative to the edge jump than that seen in the reference spectrum. The only way to fit a spectrum distorted in this way to a sum of those shown in Fig. 1 is 
to add some sulfide or metal to the assumed mix, as these species produce significant signal at the relevant energy. In general, then, over-absorption in K-edge spectra will cause thick or concentrated spots to appear chemically reduced compared to their actual states. This overabsorption effect is an example of an XRF matrix effect that depends on the chemical state as well as the composition. Similarly, in transmission, a very thick particle may exhibit a "hole" effect, leading to the same sort of errors as over-absorption in a concentrated particle in fluorescence. A possible fix for this, at the transitionmetal K-edges at least, is to map at the energies of preedge features, where the absorption for the selected transition is relatively weak.

In the hard X-ray regime, mapping usually takes long enough so that it can be done at just a few energies per sample, so it is important to choose the energies wisely. The lowest (background) energy should not be too far below the lowest-energy edge feature, as energy-dependent backgrounds and sensitivity factors could cause artifacts. Similarly, the highest energy (representing the total amount of element) should not be too high, but should be above the strongest EXAFS features. In between, it is not obvious how to choose the energies for best discrimination between species. If there are only two species to distinguish, then a third energy may be chosen to maximize the ratio of responses $Y_{1}(E) / Y_{2}(E)$ and a fourth to minimize this ratio. Maps at these energies, background-subtracted, serve as maps of the concentrations of the corresponding species, somewhat contaminated by signals from the other species. Applying Equations (3) removes this “contamination". For a larger number of species, there are no obvious answers. I have developed a tool, available through the ALS Beamline 10.3.2 website [44], which helps in the selection of energies. It works by creating simulated data sets, adding noise, and evaluating the errors caused by the noise as the user manipulates sliders representing the energies at which spectra are taken.

Another problem is the choice of species to use as references in analysis. As seen at the top of Fig. 1, the XANES spectra for different compounds having the target element in the same valence state do not always look alike. Thus, if the sample contains a species whose XANES spectrum is not close to one of the references, that species will be misinterpreted as some mixture of the other species, and that mixture will not in general have the same valence or other properties as the actual, unidentified species. Again, this problem is exacerbated in the hard X-ray realm because of the limited number of energies in the "XANES spectrum" gathered at each pixel. In STXM/TXM, where one may take data at 100 or more energies, it becomes clear whether the set of reference spectra is sufficient to account for all the spectral features found in the images.
A similar problem arises due to the polarization of the synchrotron X-ray beam. Just as single crystals of minerals are often dichroic and birefringent in the visible, creating contrast in a polarizing microscope, so too are non-cubic crystals dichroic in the X-ray. If the sample contains single crystals larger than the spatial resolution, the XANES spectrum of the crystal will not match the powder XANES spectrum used as a standard. This effect usually has little effect on qualitative valence-state images, but, in principle, it could cause errors.

\subsection{Resolution and sensitivity}

It is difficult to give categorical numbers for the resolution and trace-element sensitivity available with these methods, as every beamline is different, as is every sample. However, a look at the literature can provide some general guidance. By looking at the websites for the various light sources, one can see that the spot size available from hard X-ray microprobes varies in the range $0.1-5 \mu \mathrm{m}$. These numbers are of course subject to downward revision with time. In the soft X-ray regime, resolutions down to $15 \mathrm{~nm}$ can be obtained [45]. It should be noted that sometimes extremely high spatial resolution does not yield any advantage because, in a thick sample, the X-ray beam probes a cylindrical volume whose length is determined by the penetration depth of the X-rays, which can be many microns. A particle concentrated in the element of interest can generally be detected even if is considerably smaller than the probe beam. In that case, the particle appears as a blob whose dimensions are that of the beam. Thus, it may be possible to detect $100-\mathrm{nm}$ particles with a probe $>1 \mu \mathrm{m}$.

As for concentration of trace elements, this depends heavily on the desired resolution, the concentrations of interfering matrix elements (e.g., Fe when measuring $\mathrm{Co}$ ), the particular beamline one is using, the thickness of the sample, and whether one is measuring a diffuse area or a point particle. In the diffuse case, the relevant sensitivity figure has dimensions of ppm (equivalent to atoms $/ \mathrm{cm}^{3}$ ) or atoms $/ \mathrm{cm}^{2}$, depending on whether the sample is optically thick or thin, respectively. The reviews by Lombi and Susini [3] and by Sutton et al. [34] give some examples of limits of detection (LODs) as does the article by Twining et al. [40]. Typical LODs are in the ppm range, or, expressed as a column density, a fraction of a monolayer. However, even if the bulk average concentration is low, the element of interest may occur as "hot" particles occupying a small part of the volume, in which case they are easily detectable. For more on the sensitivity and resolution achievable at X-ray microprobes, please see other articles in this issue.

Another limit is the accuracy of valence detection and the sensitivity with which one can detect a small amount of one valence state in the presence of a large amount of another. This, too, is very system dependent. Suppose 
one is looking for a small amount of $\mathrm{Fe}^{\mathrm{O}}$ in a matrix of $\mathrm{Fe}^{3+}$ (see Fig. 1 for the relevant spectra). In that case, since $\mathrm{Fe}^{3+}$ yields little signal at an energy at which $\mathrm{Fe}^{\mathrm{O}}$ shows "brightly", it is relatively easy to detect the zerovalent $\mathrm{Fe}$ at a level of percents. If the proportions are reversed, then it becomes much more difficult to detect the minority species because there is no energy at which the minority species contributes a large per-atom signal and the majority is relatively silent. In this case, the LOD for the minority species may be up to $30 \%$. Also, as discussed above, variation of the XANES signal between different species having the same valence may compromise detection accuracy and sensitivity.

\section{Possibilities and extensions}

Just as XRF mapping has a three-dimensional tomographic extension, so too does mineral mapping [46]. Instead of a 2D detector, one may use an incident white beam and an energy-dispersive detector that picks up the diffracted beam at a fixed angle [47]. In this case, the volume being probed is defined by the intersection of the main beam and the projection back from the detector. This tomographic extension is called diffraction tomography but should not be confused with a coherentimaging method with the same name [48].

Diffractive imaging, in the sense of Chapman et al. [48], could, in principle, be used in two or three dimensions to perform chemical-state imaging. In this method, a fullfield image of the sample is made by shining a coherent beam on it and collecting a far-field diffraction pattern, which is then reconstructed by phase-retrieval methods [49]. This method promises extremely high resolution and insensitivity to vibrations. Also, the reconstruction can yield both real and imaginary parts of the refractive index, which each depend on energy and chemical species in a distinct way. Thus, performing diffractive imaging at several energies should yield chemical information. Diffractive imaging is at present done mostly in the soft X-ray range. Ptychography [50] is a related method in which a small spot is focused on the sample and a convergent-beam diffraction pattern taken, after which the spot is moved a little and another pattern taken, until the whole field is "painted" with overlapping spots. This technique is in a sense a hybrid of STXM and diffractive imaging and promises to provide the advantages of both.

The newer generation of hard X-ray microprobes features spot sizes approaching what is available in the soft X-ray. Further, the high flux densities available with undulator sources should make possible the acquisition of images at rates high enough to permit one to obtain data at many energies, thus allowing greater chemical specificity than is now practical. A new set of microprobes is becoming available for the range $1-4 \mathrm{keV}$, in which lie edges of many elements of interest that are currently hard to probe. The ability to image at resolutions smaller than the size of a bacterium will be useful in the rapidly-growing field of environmental geomicrobiology. However, caution is in order with respect to all these new methods and possibilities - radiation-induced chemistry is a known issue and could corrupt the data one wants as the data are being taken.

\section{Acknowledgements}

The operations of the Advanced Light Source at Lawrence Berkeley National Laboratory are supported by the Director, Office of Science, Office of Basic Energy Sciences, US Department of Energy under contract number DE-AC02-05CH11231. The author acknowledges the help of Ryan Ogliore, Sirine C. Fakra, Maria Chrysochoou and Brandy Toner for providing the raw material for the figures.

\section{References}

[1] S. Katz, H. Salem, J. Appl. Toxicol. 13 (1993) 217.

[2] Y. Oya-Ohta, T. Kaise, T. Ochi, Mutat. Res., Fundam. Mol. Mech. Mutagen. 357 (1996) 123.

[3] E. Lombi, J. Susini, Plant Soil 320 (2009) 1.

[4] B. Golosio, A. Somogyi, A. Simionovici, P. Bleuet, J. Susini, L. Lemelle, Appl. Phys. Lett. 84 (2004) 2199.

[5] L. Vincze, B. Vekemans, F. Brenker, G. Falkenberg, K. Rickers, A. Somogyi, M. Kersten, F. Adams, Anal. Chem. 76 (2004) 6786.

[6] B. Vekemans, L. Vincze, F. Brenker, F. Adams, J. Anal. At. Spectrom. 19 (2004) 1302.

[7] A. Manceau, N. Tamura, M.A. Marcus, A.A. MacDowell, R.S. Celestre, R.E. Sublett, G. Sposito, H.A. Padmore, Am. Mineral. 87 (2002) 1494.

[8] E. Dooryhée, M. Anne, I. Bardiès, J. Hodeau, P. Martinetto, S. Rondot, J. Salomon, G. Vaughan, P. Walter, Appl. Phys. A: Mater. Sci. Process. 81 (2005) 663.

[9] J. Kinney, Q. Johnson, M. Nichols, U. Bonse, R. Nusshardt, Appl. Opt. 25 (1986) 4583.

[10] J. Kinney, Q. Johnson, R. Saroyan, M. Nichols, U. Bonse, R. Nusshardt, R. Pahl, Rev. Sci. Instrum. 59 (1988) 196.

[11] I.J. Pickering, R.C. Prince, D.E. Salt, G.N. George, Proc. Natl. Acad. Sci. USA 97 (2000) 10717.

[12] R.C. Ogliore, A.L. Butterworth, S. Fakra, D.R. Frank, Z. Gainsforth, M.A. Marcus, A.J. Westphal, 39th Lunar Planet. Sci. Conf., League City, Texas, USA, 2008.

[13] B.M. Toner, S.C. Fakra, S.J. Manganini, C.M. Santelli, M.A. Marcus, J.W. Moffett, O. Rouxel, C.R. German, K.J. Edwards, Nat. Geosci. 2 (2009) 197.

[14] C. Schroer, M. Kuhlmann, T. Günzler, B. Lengeler, M. Richwin, B. Griesebock, D. Lützenkirchen-Hecht, R. Frahm, E. Ziegler, A. Mashayekhi, Appl. Phys. Lett. 82 (2003) 3360.

[15] S. Myneni, in: P.A. Fenter, M. Rivers, N.C. Sturchio, S.R. Sutton (Editors), Reviews in Mineralogy and Geochemistry, Mineral Soc. America, Washington, DC, USA, 2002, pp. 485-579.

[16] J. Lawrence, G. Swerhone, G. Leppard, T. Araki, X. Zhang, M. West, A. Hitchcock, Appl. Environ. Microbiol. 69 (2003) 5543.

[17] C. Chan, G. De Stasio, S. Welch, M. Girasole, B. Frazer, M. Nesterova, S. Fakra, J. Banfield, Science (Washington, DC) 303 (2004) 1656.

[18] X. Zhang, R. Balhorn, J. Mazrimas, J. Kirz, J. Struct. Biol. 116 (1996) 335. 
[19] E. Bulska, I. Wysocka, M. Wierzbicka, K. Proost, K. Janssens, G. Falkenberg, Anal. Chem. 78 (2006) 7616.

[20] S.R. Sutton, S. Bajt, J. Delaney, D. Schulze, T. Tokunaga, Rev. Sci. Instrum. 66 (1995) 1464

[21] I.J. Pickering, L. Gumaelius, H.H. Harris, R.C. Prince, G. Hirsch, J.A. Banks, D.E. Salt, G.N. George, Environ. Sci. Technol. 40 (2006) 5010 .

[22] I.J. Pickering, E.Y. Sneeden, R.C. Prince, E. Block, H.H. Harris, G. Hirsch, G.N. George, Biochemistry 48 (2009) 6846.

[23] I.J. Pickering, G. Hirsch, R.C. Prince, E.Y. Sneeden, D.E. Salt, G.N. George, J. Synchrotron Radiat. 10 (2003) 289.

[24] J. Thieme, I. McNult, S. Vogt, A.D. Paterson, Environ. Sci. Technol. 41 (2007) 6885.

[25] J.L. Freeman, L.H. Zhang, M.A. Marcus, S. Fakra, S.P. McGrath, E.A.H. Pilon-Smits, Plant Physiol 142 (2006) 124.

[26] M. Chrysochoou, S. Fakra, M. Marcus, D. Moon, D. Dermatas, Environ. Sci. Technol. 43 (2009) 5461.

[27] M. Chrysochoou, D. Dermatas, 11th Int. Conf. Environ. Sci. Technol., Chania, Greece, 2009.

[28] M. Andreani, O. Grauby, A. Baronnet, M. Muñoz, Eur. J. Mineral. 20 (2008) 159

[29] M. Muñoz, V. De Andrade, O. Vidal, E. Lewin, S. Pascarelli, J. Susini, Geochem. Geophys. Geosyst. 7 (2006) Q11020.

[30] J. Foriel, P. Philippot, J. Susini, P. Dumas, A. Somogyi, M. Salomé, H. Khodja, B. Menez, Y. Fouquet, D. Moreira, Geochim. Cosmochim. Acta 68 (2004) 1561.

[31] L.L. Oram, D.G. Strawn, M.A. Marcus, S.C. Fakra, G. Moller, Environ. Sci. Technol. 42 (2008) 6830

[32] A.L. Ryser, D.G. Strawn, M.A. Marcus, S. Fakra, J.L. JohnsonMaynard, G. Moller, Environ. Sci. Technol. 40 (2006) 462.

[33] V.A. Solé, E. Papillon, M. Cotte, P. Walter, J. Susini, Spectrochim. Acta, Part B 62 (2007) 63.

[34] S.R. Sutton, P.M. Bertsch, M. Newville, M. Rivers, A. Lanzirotti, P. Eng, in: P.A. Fenter, M.L. Rivers, N.C. Sturchio, S.R. Sutton (Editors), Applications of Synchrotron Radiation in Low-Temperature Geochemistry and Environmental Science, Mineralogical Society of America, Washington, DC, USA, 2002, pp. 429-478.

[35] J. Goulon, C. Goulon-Ginet, R. Cortes, J.M. Dubois, J. Physique 42 (1981) 539.

[36] M. Lerotic, C. Jacobsen, T. Schäfer, S. Vogt, Ultramicroscopy 100 (2004) 35 .
[37] A. Manceau, M.A. Marcus, N. Tamura, in: P.A. Fenter, M.L. Rivers, N.C. Sturchio, S.R. Sutton (Editors), Applications of Synchrotron Radiation in Low-Temperature Geochemistry and Environmental Science, Mineralogical Society of America, Washington, DC, USA, 2002, pp. 341-428.

[38] M.A. Marcus, A.A. MacDowell, R. Celestre, A. Manceau, T. Miller, H.A. Padmore, R.E. Sublett, J. Synchrotron Radiat. 11 (2004) 239.

[39] I. McNulty, D. Paterson, J. Arko, M. Erdmann, S. Frigo, K. Goetze, P. Ilinski, N. Krapf, T. Mooney, C. Retch, J. Phys. IV France 104 (2003) 11.

[40] B. Twining, S. Baines, N. Fisher, J. Maser, S. Vogt, C. Jacobsen, A. Tovar-Sanchez, S. Sanudo-Wilhelmy, Anal. Chem. 75 (2003) 3806.

[41] S. Heim, P. Guttmann, S. Rehbein, S. Werner, G. Schneider, J. Physics: Conf. Ser. (2009).

[42] <http://unicorn.mcmaster.ca/aXis2000.html> (Access checked December 2009).

[43] < http://ssrl.slac.stanford.edu/beamlines/bl2-3/> (Access checked December 2009).

[44] <http://xraysweb.lbl.gov/uxas/Beamline/Software/Utilities/ chem\%20map\%20error\%20estimator.zip> (Access checked December 2009).

[45] K. Jefimovs, J. Vila-Comamala, T. Pilvi, J. Raabe, M. Ritala, C. David, Phys. Rev. Lett. 99 (2007) 264801.

[46] P. Bleuet, E. Welcomme, E. Dooryhée, J. Susini, J. Hodeau, P. Walter, Nat. Mater. 7 (2008) 468

[47] C. Hall, P. Barnes, J. Cockcroft, S. Colston, D. Häusermann, S. Jacques, A. Jupe, M. Kunz, Nucl. Instrum. Methods Phys. Res., Sect. B 140 (1998) 253.

[48] H. Chapman, A. Barty, S. Marchesini, A. Noy, S. Hau-Riege, C. Cui, M. Howells, R. Rosen, H. He, J. Spence, J. Opt. Soc. Am. A 23 (2006) 1179

[49] A. Scherz, D. Zhu, R. Rick, W. Schlotter, S. Roy, J. Lüning, J. Stöhr, Phys. Rev. Lett. 101 (2008) 76101.

[50] J. Rodenburg, A. Hurst, A. Cullis, B. Dobson, F. Pfeiffer, O. Bunk, C. David, K. Jefimovs, I. Johnson, Phys. Rev. Lett. 98 (2007) 34801.

[51] M.A. Marcus, A.J. Westphal, S.C. Fakra, J. Synchrotron Radiat. 15 (2008) 463. 